\title{
Single-Ensemble-Based Eigen-Processing Methods for Color Flow Imaging-Part I. The Hankel-SVD Filter
}

\author{
Alfred C. H. Yu, Member, IEEE, and Richard S. C. Cobbold, Life Member, IEEE
}

\begin{abstract}
Because of their adaptability to the slow-time signal contents, eigen-based filters have shown potential in improving the flow detection performance of color flow images. This paper proposes a new eigen-based filter called the Hankel-SVD filter that is intended to process each slowtime ensemble individually. The new filter is derived using the notion of principal Hankel component analysis, and it achieves clutter suppression by retaining only the principal components whose order is greater than the clutter eigenspace dimension estimated from a frequency-based analysis algorithm. To assess its efficacy, the Hankel-SVD filter was first applied to synthetic slow-time data (ensemble size: 10) simulated from two different sets of flow parameters that model: 1) arterial imaging (blood velocity: 0 to $38.5 \mathrm{~cm} / \mathrm{s}$, tissue motion: up to $2 \mathrm{~mm} / \mathrm{s}$, transmit frequency: $5 \mathrm{MHz}$, pulse repetition period: $0.4 \mathrm{~ms}$ ) and 2) deep vessel imaging (blood velocity: 0 to $19.2 \mathrm{~cm} / \mathrm{s}$, tissue motion: up to $2 \mathrm{~cm} / \mathrm{s}$, transmit frequency: $2 \mathrm{MHz}$, pulse repetition period: $2.0 \mathrm{~ms}$ ). In the simulation analysis, the post-filter clutterto-blood signal ratio (CBR) was computed as a function of blood velocity. Results show that for the same effective stopband size $(50 \mathrm{~Hz})$, the Hankel-SVD filter has a narrower transition region in the post-filter CBR curve than that of another type of adaptive filter called the clutterdownmixing filter. The practical efficacy of the proposed filter was tested by application to in vivo color flow data obtained from the human carotid arteries (transmit frequency: $4 \mathrm{MHz}$, pulse repetition period: $0.333 \mathrm{~ms}$, ensemble size: 10). The resulting power images show that the Hankel-SVD filter can better distinguish between blood and movingtissue regions (about $9 \mathrm{~dB}$ separation in power) than the clutter-downmixing filter and a fixed-rank multi-ensemblebased eigen-filter (which showed a 2 to $3 \mathrm{~dB}$ separation).
\end{abstract}

\section{INTRODUCTION}

$\mathrm{I}_{\mathrm{A}}^{\mathrm{N}}$ $\mathrm{N}$ ultrasound color-flow imaging, the computation of flow estimates for each sample volume (or map pixel location) within the imaging view is often regarded as a nontrivial task from a signal processing perspective. The difficulty of this computational operation is particularly leveraged by the presence of high-energy, low-frequency clutter (originating from tissue reverberations and beam sidelobe leakages) that masks out the desired blood echoes

Manuscript received January 17, 2007; accepted January 8, 2008. A. C. H. Yu is with the University of Hong Kong, Department of Electrical and Electronic Engineering, Pokfulam, Hong Kong.

R. S. C. Cobbold is with the University of Toronto, Institute of Biomaterials and Biomedical Engineering, Toronto, Ontario, Canada (e-mail: cobbold@ecf.utoronto.ca).

Digital Object Identifier 10.1109/TUFFC.2008.682 in the acquired slow-time data. Hence, as one of the first steps in color flow signal processing, a highpass filtering procedure is applied to each raw slow-time ensemble (i.e., the received signal samples after fast-time demodulation and range gating at a nominal depth) so that the clutter can be suppressed before the computation of blood flow estimates. Given that the slow-time ensemble size is typically less than 20 samples owing to real-time imaging constraints, such filtering can be more effectively performed using time-variant filters that have a less significant transient response. As reviewed by Bjaerum et al. [1], the projection-initialized infinite impulse response (IIR) filter and the polynomial regression filter are two particular types of time-variant filters that are useful for slowtime clutter suppression. However, since these filters cannot adaptively position their stopband locations based on the signal contents, they inherently require a larger stopband to suppress nonzero-frequency clutter that may arise when significant tissue motion is present over the data acquisition time frame. As a result, they may concomitantly suppress a substantial portion of the blood echoes, and in turn the sensitivity of flow detection may be decreased.

\section{A. Review of Existing Adaptive Filter Designs}

To improve the suppression of clutter arising from moving tissues, some studies have considered the use of filtering methods that can adapt its stopband to the clutter spectral characteristics. For instance, Thomas and Hall [2] as well as Brands et al. [3] have proposed an approach that first involves the downmixing of the slow-time signal with the mean clutter frequency before the highpass filtering operation. This downmixing step is intended to shift the clutter spectral components toward zero frequency, thereby making it possible for the slow-time clutter to be suppressed without widening the filter stopband. Nevertheless, as pointed out elsewhere [4], this clutter-downmixing approach can only be treated as a partially adaptive filtering strategy because its performance inherently depends on the choice of the highpass filter.

Over the past decade, an increasing amount of effort has been devoted to using eigen-based methods (also known as principal component analysis) for color flow signal processing. These eigen-processing methods generally work by decomposing slow-time ensembles into a series of orthogonal bases (as opposed to fixed bases like the Fourier expansion) and identifying the ones that are related to blood 
echoes. Since the orthogonal expansion is computed based on the ensemble contents, eigen-based signal processing strategies have the theoretical advantage of being able to adapt to the slow-time signal characteristics, and thus they have potential in suppressing nonstationary clutter while preserving the blood echoes in the slow-time data. In the context of clutter suppression, eigen-processing methods appear to be first considered by Ledoux et al. [5], who proposed to remove stationary clutter from an array of slowtime ensembles along the same line-of-sight by eliminating the most dominant components in the singular value decomposition (SVD) of the ensemble array. Bjaerum et al. [6] then made use of this subspace-based filtering concept to develop an eigen-regression filter that is based on an eigen-decomposition of the slow-time correlation statistics. Around the same time, Kruse and Ferrara [7] designed a similar filtering strategy for use in high-frequency sweptscan-based flow imaging, while Kargel et al. [8] applied the same approach to their strain-flow hybrid imaging mode. On the other hand, Gallippi et al. [9], [10] evaluated the use of an independent component analysis framework to suppress clutter in their acoustic-radiation-force imaging studies.

\section{B. Motivations of Study}

Despite its adaptability to the slow-time signal contents, in practice the eigen-regression filter is often faced with two types of implementation challenges. First, consistent estimation of slow-time correlation matrix normally requires multiple signal ensembles with clutter characteristics that are statistically stationary. In the existing eigen-based filter designs, this estimation procedure is often carried out through ensemble averaging of the slow-time signal ensembles along the same line-of-sight or interleaved pulse firing region (with the inherent assumption that the clutter statistics are stationary over the depth of view). Such an approach is generally effective in microvasculature imaging studies where the imaging depths are typically a few millimeters, but it is less likely to be valid for arterial imaging studies with centimeter-scale imaging depths over which the extent of tissue motion may not be coherent. The other limitation of the eigen-regression filter is that its efficacy is rather sensitive to the choice of the clutter eigen-space dimension (i.e., the number of principal components corresponding to clutter) owing to the filter's adaptive nature. As such, it is necessary to develop an effective algorithm for this filter to select the clutter eigen-space dimension.

In the first of this two-part paper series, we present a novel eigen-based clutter filter design that does not require the use of multiple slow-time ensembles to perform the orthogonal decomposition. This filter, which we refer to as the Hankel-SVD filter, works by exploiting the eigenspace properties of a matrix form known as the Hankel matrix whose entries are constant along the reverse diagonals and whose orthogonal expansion can be found from an SVD analysis. To formulate discussion on the HankelSVD filter, the rest of this paper is organized as follows.
Section II presents the theoretical principles of the HankelSVD filter and describes a frequency-based algorithm for estimating the clutter eigen-space dimension. Section III then details the slow-time signal synthesis method used to analyze the new filter's flow detection performance, and the corresponding results are discussed in Section IV. An in vivo case study on the use of the Hankel-SVD filter in suppressing slow-time clutter is presented in Section V. Some concluding remarks are provided in Section VI.

\section{Principles of the HANKEL-SVD Filter}

Like existing eigen-regression filters, the proposed Hankel-SVD filter attempts to decompose the slow-time signal as a sum of orthogonal basis functions. However, instead of relying on multiple ensembles of slow-time signal to estimate the basis functions, the Hankel-SVD approach computes the orthogonal bases through an SVD of a Hankel data matrix (which has constant entries along its reverse diagonals) created from the slow-time signal of individual sample volumes. For a given slow-time ensemble $\mathbf{x}$ with $N_{D}$ samples, the signal approximation obtained from the Hankel-SVD approach can be expressed as

$$
\mathbf{x}=\left[x(0), x(1), \ldots, x\left(N_{D}-1\right)\right]^{T} \approx \sum_{k=1}^{P} \gamma_{k} \boldsymbol{\varphi}_{k}
$$

In the above, $P$ is the number of orthogonal bases in the Hankel component approximation (with $P<N_{D}$ ), while $\gamma_{k}$ and $\varphi_{k}$ are, respectively, the $k$ th expansion coefficient and orthonormal basis vectors that satisfies the following orthogonality relation:

$$
E\left\{\boldsymbol{\varphi}_{k}^{H} \boldsymbol{\varphi}_{l}\right\}=\left\{\begin{array}{ll}
1 & (k=l) \\
0 & (k \neq l)
\end{array} .\right.
$$

This formulation is based on a principal Hankel component analysis framework that is originally used in timeseries analysis and array processing [11]. Such an approach should be distinguished from the SVD analysis framework reported by Ledoux et al. [5], who used multiple slow-time signal ensembles to compute the orthogonal bases.

\section{A. Computation of Orthogonal Bases}

An overview of the proposed single-ensemble-based eigen-filtering strategy is depicted in Fig. 1. This filtering strategy begins with the creation of a Hankel data matrix by dividing a slow-time ensemble into partially overlapping segments and rearranging them into the following array ${ }^{1}$ :

$$
\mathbf{A}=\left[\begin{array}{cccc}
x(0) & x(1) & \cdots & x\left(N_{D}-P\right) \\
x(1) & x(2) & \cdots & x\left(N_{D}-P+1\right) \\
\vdots & \vdots & \ddots & \vdots \\
x(P-1) & x(P) & \cdots & x\left(N_{D}-1\right)
\end{array}\right]_{P \times\left(N_{D}-P+1\right)}
$$

\footnotetext{
${ }^{1}$ The $(i, j)$ th Hankel matrix entry takes on the $(i+j)$ th sample in the signal ensemble.
} 


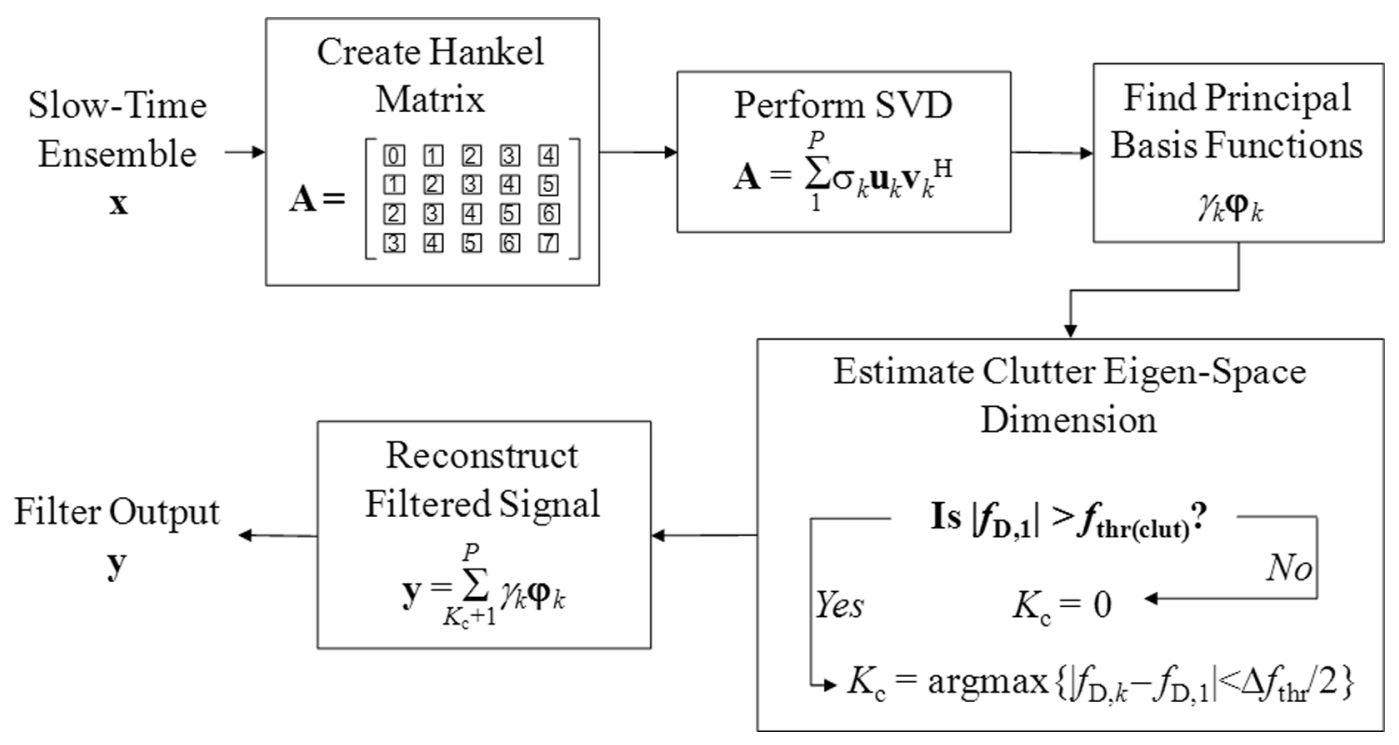

Fig. 1. Flow diagram of the proposed Hankel-SVD filter. During operation, the filter is applied to the slow-time ensemble of each sample volume.

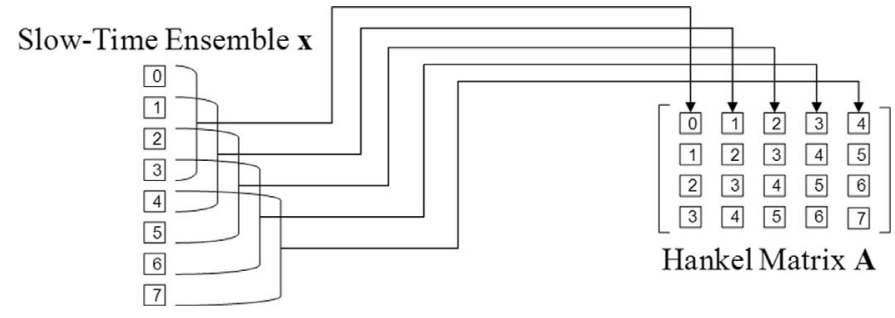

Fig. 2. Illustration of how the Hankel matrix $\mathbf{A}$ is constructed for the case where the slow-time ensemble size is eight $\left(N_{D}=8\right)$ and the dimension parameters is four $(P=4)$.

where $P$ must satisfy the relation $P \leq \operatorname{ceil}\left(N_{D} / 2\right)$, i.e., the smallest integer greater than or equal to $N_{D} / 2$. As such, the relation $P \leq N_{D}-P+1$ always holds, and the matrix A has a rank (i.e., the number of independent rows) that is equal to $P$ with a largest possible rank of $\operatorname{ceil}\left(N_{D} / 2\right)$. An example of the entire matrix formation process is shown in Fig. 2. Note that, for this matrix, statistical stationarity of the samples in the signal vector is inherently assumed.

To compute the $P$ orthogonal components in the Hankel component approximation, we can factor the Hankel data matrix into the following sum of $P$ orthogonal, rank-one matrix components via the SVD:

$$
\mathbf{A}=\sum_{k=1}^{P} \mathbf{A}_{k}=\sum_{k=1}^{P} \sigma_{k} \mathbf{u}_{k} \mathbf{v}_{k}^{H} .
$$

In this expression, $\mathbf{A}_{k}$ is the $k$ th rank-one Hankel component in the decomposition, while $\sigma_{k}, \mathbf{u}_{k}$, and $\mathbf{v}_{k}$ are correspondingly the singular value, left singular vector (with dimension $P$ ), and right singular vector (with dimension $N_{D}-P+1$ ) of $\mathbf{A}_{k}$. Note that the $P$ singular values in (3) are ordered from largest to smallest by definition, and hence $\mathbf{A}_{k}$ can be considered as the $k$ th-order principal Hankel component. From these principal Hankel components, it is possible to reconstruct the orthogonal basis vectors $\gamma_{k} \boldsymbol{\varphi}_{k}$ as seen in (1a). One particular way to perform this reconstruction process, as originally described by Poon et al. [12], is to sum and average the matrix elements along the reverse diagonals of $\mathbf{A}_{k}$ because the matrix more or less maintains a constant reverse-diagonal structure. Such an approach is used by the proposed filtering method.

\section{B. Estimation of Clutter Eigen-Space Dimension}

Because the aim of the Hankel-SVD filter is to suppress clutter in the slow-time signal, it is necessary to determine whether a principal Hankel component $\mathbf{A}_{k}$ is part of the clutter eigen-space. In general, there are two types of approaches to carry out this analysis. First, given that clutter often has higher energy than blood echoes and white noise, a principal Hankel component can be considered as being part of the clutter eigen-space if its singular value magnitude $\sigma_{k}$ is larger than a given value. Such way of estimating the clutter eigen-space dimension is similar to the eigenvalue analysis algorithms reported previously in a few eigen-filter designs [7], [13]. Alternatively, since clutter generally consists of low-frequency contents, it is possible to identify a clutter eigen-space component based on the frequency contents of each orthonormal basis vector $\varphi_{k}$. This latter approach is adopted in the Hankel-SVD filter to determine the clutter eigen-space dimension because the approach can be implemented in a way that gives filtering characteristics similar to the stopband of a conventional bandpass filter.

The clutter eigen-space analysis algorithm used by the Hankel-SVD filter is illustrated in Fig. 3. This algorithm is fundamentally based on two assumptions: 1) the slowtime clutter is contained in the more dominant Hankel components (i.e., ones with larger singular values); 2) the blood flow component of the slow-time signal is contained in the Hankel components with high Doppler frequen- 

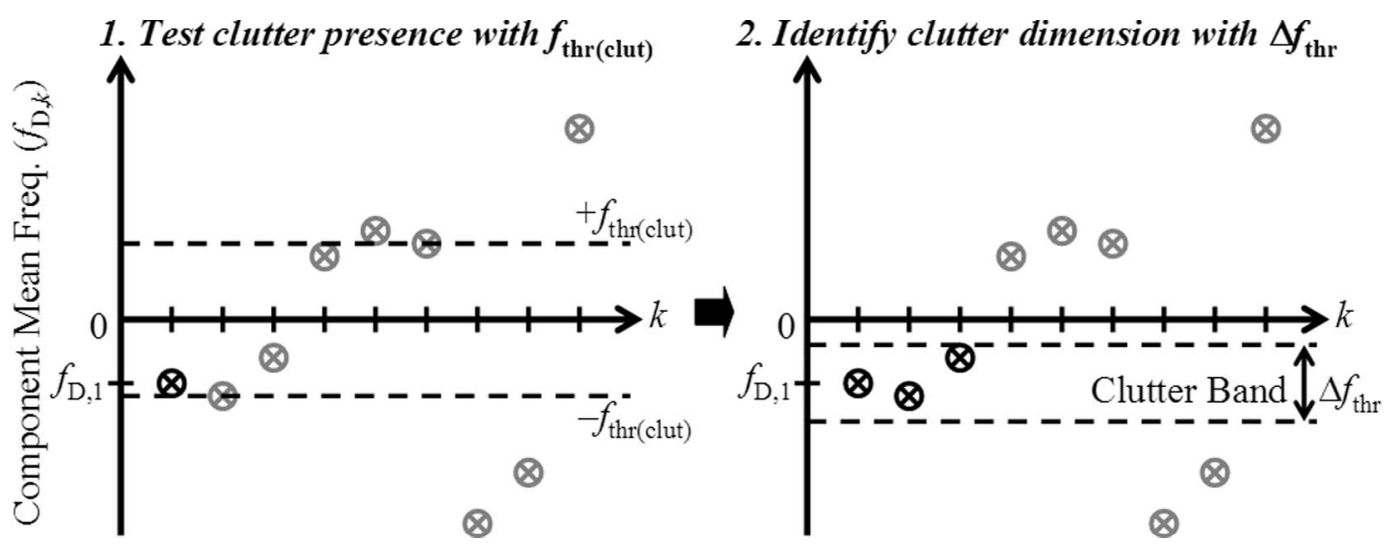

Fig. 3. Steps used by the Hankel-SVD filter to estimate the clutter eigen-space dimension. First (left), the clutter characteristics are examined by testing whether the most dominant component has a mean frequency (marked as black $\otimes$ ) less than a threshold $f_{\text {thr(clut). After that }}$ (right), the clutter dimension is determined by finding higher component order with a mean frequency estimate inside the clutter band $\Delta f_{\text {thr }}$ (in this example, the clutter dimension is equal to three).

cies. Specifically, the analysis begins with an assessment of whether clutter is present in the slow-time ensemble by finding whether the most dominant Hankel component has a mean frequency $f_{D, 1}$ that is less than a spectral threshold $f_{\text {thr(clut) }}$. If clutter is considered to be present, then the mean frequency of the other Hankel components is also estimated. Note that, by using the well-known lagone autocorrelator [14], the mean Doppler frequency $f_{D, k}$ of a Hankel component can be found from the orthonormal basis vector $\varphi_{k}$ as follows:

$$
f_{D, k}=\frac{1}{2 \pi T_{\mathrm{PRI}}} \arg \left[\sum_{n=1}^{N_{D}-1} \varphi_{k}(n) \varphi_{k}^{*}(n-1)\right],
$$

where $T_{\mathrm{PRI}}$ is the pulse repetition interval (i.e., the slowtime sampling interval). From these estimates, the clutter eigen-space dimension is found by searching for the largest component order whose mean frequency falls within a clutter band $\Delta f_{\text {thr }}$ that is centered at $f_{D, 1}$ (as shown in Fig. 3). Consequently, the estimated clutter dimension $K_{c}$ can be expressed as (5) (see next page). Once the clutter dimension has been estimated, the filtered slow-time signal $\mathbf{y}$ can be found as follows by summing the orthogonal basis functions beyond the estimated clutter dimension:

$$
\mathbf{y}=\left[y(0), y(1), \ldots, y\left(N_{D}-1\right)\right]^{T}=\sum_{k=K_{C}+1}^{P} \gamma_{k} \boldsymbol{\varphi}_{k} .
$$

It is worth noting that the average power of the filtered slow-time signal can be actually estimated from the singular values of the principal Hankel components beyond the clutter dimension. In particular, since the squared sum of singular values is equal to the Frobenius matrix norm (i.e., the squared sum of matrix entries), the post-filter signal power $\rho_{\mathbf{y}}$ can be estimated as

$$
\rho_{\mathbf{y}}=\sum_{k=K_{C}+1}^{P} \frac{\sigma_{k}^{2}}{P\left(N_{D}-P+1\right)},
$$

where the normalization factor in the denominator stems from the fact that each Hankel component $\mathbf{A}_{k}$ has $P\left(N_{D}-\right.$ $P+1)$ entries.

\section{Relationship to Existing Eigen-Filters}

The theoretical formulation of the Hankel-SVD filter can actually be linked to existing eigen-regression filters. In particular, the Hankel-SVD approach can be considered as a signal-domain formulation of a modified eigen-regression filter that involves data smoothing when computing the correlation matrix. This relationship can be seen by first noting that the Hankel matrix A in the Hankel-SVD filter can be converted into the following $P \times P$ correlation matrix:

$$
\begin{aligned}
& \mathbf{R}_{\mathbf{x}} \approx \mathbf{A A}^{H}= \\
& {\left[\begin{array}{cccc}
R_{0}(0) & R_{1}(-1) & \cdots & R_{P-1}(-P+1) \\
R_{0}(1) & R_{1}(0) & \cdots & R_{P-1}(-P+2) \\
\vdots & \vdots & \ddots & \vdots \\
R_{0}(P-1) & R_{1}(P-2) & \cdots & R_{P-1}(0)
\end{array}\right]_{P \times P}} \\
& \text { for } R_{k}(l)=\sum_{n=k+l}^{N_{D}-P+k+l} x(n) x^{*}(n-l),
\end{aligned}
$$

where $R_{k}(l)$ is the smoothed autocorrelation estimate for the $l$ th lag and is computed by summing the single-sample correlation values over a window of $N_{D}-P+1$ samples (with $k$ being the first sample index in the window when the lag is zero). From the properties of the SVD, it is well known that the eigen-decomposition of this smoothed correlation matrix is related to the SVD of the Hankel data matrix as follows:

$$
\mathbf{A}=\sum_{k=1}^{P} \sigma_{k} \mathbf{u}_{k} \mathbf{v}_{k}^{H} \Leftrightarrow \mathbf{A} \mathbf{A}^{H}=\sum_{k=1}^{P} \sigma_{k}^{2} \mathbf{u}_{k} \mathbf{u}_{k}^{H},
$$

where the eigenvalues and eigenvectors of $\mathbf{A} \mathbf{A}^{H}$ are, respectively, the squared singular values and the left singular vectors of A. Based on such property, the Hankel-SVD 


$$
K_{c}= \begin{cases}0 & , \text { for }\left|f_{D, 1}\right|>f_{\text {thr(clut })} \\ \underset{k}{\arg \max }\left\{\left|f_{D, k}-f_{D, 1}\right|<\Delta f_{\text {thr }} / 2\right\} & , \text { for }\left|f_{D, 1}\right|<f_{\text {thr(clut })}\end{cases}
$$

filter can therefore be seen as being similar to an eigenregression filter that computes orthogonal bases from a smoothed correlation matrix.

\section{Computational Considerations}

As can be expected from its formulation, the computation load of the Hankel-SVD filter is rather high as compared with that for nonadaptive filters like the IIR filter. In particular, this new filtering strategy requires the computation of an SVD on the Hankel matrix formed from each slow-time signal, and after the SVD is computed, a series of vector operations is needed to reconstruct the principal Hankel components. Indeed, as shown elsewhere (see Table 2-2 in [15]), the application of this filter to each slow-time signal would need at least on the order of $P^{3}$ floating point operations when $P$ is equal to $N_{D} / 2$. Nevertheless, since $N_{D}$ is often kept below 20 samples in color flow imaging, the additional burden needed by the HankelSVD filter is less substantial. Also, by taking advantage of the persymmetric structure of the Hankel data matrix, it is possible to perform the SVD with fewer floating point operations via the use of more efficient algorithms (see [16] for a general reference).

\section{Simulation Method}

To facilitate analysis of the Hankel-SVD filter's performance, a signal synthesis model was first developed to generate slow-time ensembles with various spectral characteristics. In this model, the clutter and blood components of the slow-time signal were separately generated using two different approaches. Such a hybrid synthesis approach is physically justified by recognizing that tissue and blood scatterers follow different movement mechanisms: Tissues tend to move in a quasicyclic pattern while blood scatterers simply traverse through the sample volume. Based on these separate mechanisms, the slow-time clutter was synthesized as sampled data with phase modulation features (as described in [17]), while the blood echoes were generated by feeding complex Gaussian noise through a linear filter whose impulse response corresponds to the slow-time signal for a single moving scatterer (as originally proposed in $[18])$.

\section{A. Synthesis Procedure}

1. Clutter Simulation: An overview of our slow-time signal synthesis procedure is illustrated in Fig. 4. One of the major components in the synthesis procedure is the generation of the clutter waveform in the slow-time signal.

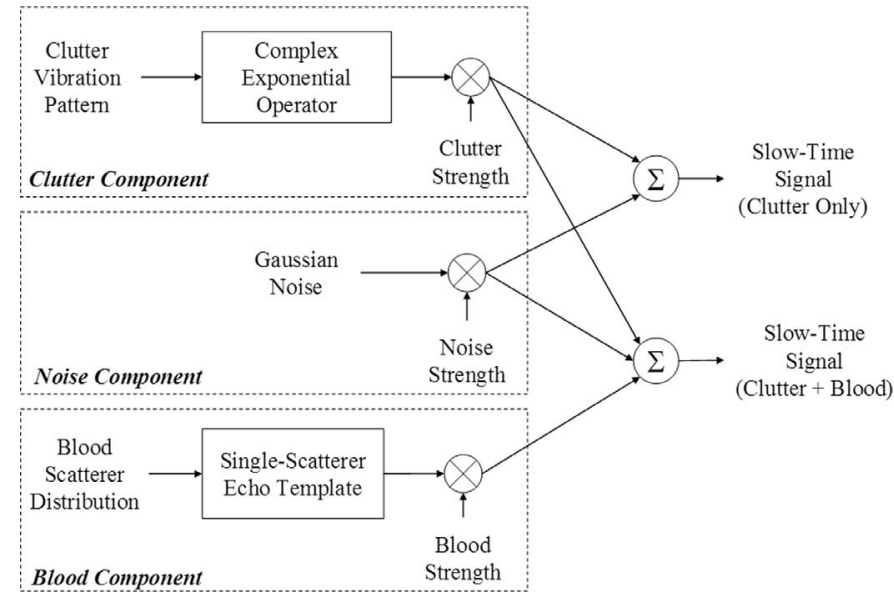

Fig. 4. System-level schematic of the slow-time signal synthesis approach. In this study, the single-scatterer blood echo template carried the form of a Gaussian-shaped complex sinusoid, while the clutter vibration pattern was a single-tone waveform.

As shown in the Appendix, this process begins by defining a clutter vibration pattern $\phi_{c}(n)$ to represent the instantaneous phase of the slow-time clutter. For our study, the vibration pattern was modeled as a sinusoid whose frequency and peak amplitude were set based on the specified clutter parameters. The clutter waveform template $h_{c}(n)$ was then obtained by setting the vibration pattern as the phase argument of a complex exponential operator, and thus the $n$th sample of this template can be expressed as

$$
h_{c}(n)=\exp \left\{j \phi_{c}(n)\right\}=\exp \left\{j \phi_{c, \max } \sin \left(2 \pi f_{\mathrm{vib}} n T_{\mathrm{PRI}}\right)\right\} \text {. }
$$

In the above, $f_{\text {vib }}$ is the vibration frequency of the clutter vibration pattern, and $\phi_{c, \max }$ is the maximum instantaneous clutter phase that equals to the following (see Appendix):

$$
\phi_{c, \text { max }}=\frac{2 f_{o} v_{c, \max } \cos \theta_{c}}{c_{o} f_{\mathrm{vib}}}
$$

where $v_{c, \max }$ is the maximum tissue velocity, $\theta_{c}$ is the beam-tissue angle, $f_{o}$ is the ultrasound frequency, and $c_{o}$ is the acoustic propagation speed. Once the clutter waveform template is defined, it is multiplied with a complex Gaussian random number (of zero mean and unit variance) to model the random magnitude and phase of coherent tissue scatterers within the sample volume. Note that this clutter simulation approach inherently assumes constant characteristics for coherent tissue scatterers throughout the ensemble period. As such, the data samples in the simulated clutter would not exhibit signal decorrelation characteristics that may arise from the cyclic movement of tissue scatterers into and out of the sample volume. 
2. Blood Signal Simulation: Another major step in the signal synthesis process is generation of the blood component in the slow-time signal. This process first involves the creation of an echo template to model the Doppler signal originating from a single moving scatterer. The echo template used in this study was defined as a Gaussianenveloped complex sinusoid whose modulating frequency corresponds to the mean Doppler frequency calculated from the Doppler equation and the specified flow velocity. For a given modulating frequency $f_{D(b)}$ and a temporal width parameter $T_{\text {width }}$, the $n$th sample of the blood echo template can be expressed as follows (see Appendix):

$$
h_{b}(n)=\exp \left\{-\left(n T_{\mathrm{PRI}} / T_{\mathrm{width}}\right)^{2}\right\} \cdot \exp \left\{j 2 \pi f_{D(b)} n T_{\mathrm{PRI}}\right\} \text {. }
$$

To account for the transit time broadening effects of blood scatterers [19], the temporal width of the blood echo template was defined as a function of blood flow velocity. In particular, for large beam-flow angles, it can be shown that the temporal width parameter is proportional to the following relation:

$$
T_{\text {width }} \propto \frac{1}{\beta_{b}} \propto \frac{c_{o} F_{\text {num }}}{2 v_{b} f_{o} \sin \theta_{b}},
$$

where $\beta_{b}$ is the spectral broadening bandwidth (assumed to be the $-20 \mathrm{~dB}$ bandwidth in the simulations), $v_{b}$ is the blood velocity, $F_{\text {num }}$ is the F-number of the transducer, and $\theta_{b}$ is the beam-flow angle. Once the single-scatterer echo template was defined, the blood signal for multiple scatterers was then synthesized by convolving complex Gaussian random samples (of zero mean and unit variance) with the template. Note that, during the simulations, the transient samples in the convolution were discarded to maintain statistical uniformity in the synthesized data. As well, multiple realizations of the blood signal were generated at once by convolving a large number of random samples with the single-scatterer echo template.

3. Slow-Time Signal Generation: To synthesize slowtime ensembles, the amplitudes of the simulated clutter and blood signals were first scaled according to the relative clutter and blood scattering strengths. After that, the scaled components were summed together along with white noise samples that were generated separately. For analysis purpose, the clutter-only slow-time signal (i.e., the signal for a sample volume with no blood flow) was also obtained by summing only the scaled clutter components and white noise samples. The $n$th sample of the synthesized slow-time signal and its clutter-only counterpart can be expressed as

$$
\begin{gathered}
x(n)=\kappa_{c}\left[g_{c} h_{c}(n)\right]+\kappa_{b}\left[g_{b}(n) * h_{b}(n)\right]+\kappa_{w} w(n), \\
x_{c}(n)=\kappa_{c}\left[g_{c} h_{c}(n)\right]+\kappa_{w} w(n)
\end{gathered}
$$

where $g_{c}$ and $g_{b}(n)$ are, respectively, the complex Gaussian random samples used to model the tissue and blood scatterer distributions; also, $\kappa_{c}, \kappa_{b}$, and $\kappa_{w}$, respectively, denote the amplitude scaling coefficients for clutter, blood echoes, and white noise. In the simulations, the relative strengths between the amplitude scaling coefficients were characterized according to the clutter-to-blood signal ratio (CBR) and the blood-signal-to-noise ratio (BSNR), which in turn are defined by

$$
\begin{aligned}
\mathrm{CBR} & =20 \log _{10}\left(\kappa_{c} / \kappa_{b}\right), \\
\mathrm{BSNR} & =20 \log _{10}\left(\kappa_{b} / \kappa_{w}\right) .
\end{aligned}
$$

Note that, before using the signals synthesized from (14a) and (14b) for filter performance analysis, they were quantized to a certain number of bits as set forth by the specified dynamic range, and they were divided into nonoverlapping segments to yield multiple signal realizations.

\section{B. Filter Analysis Protocol}

To assess the efficacy of the Hankel-SVD filter, various sets of slow-time ensembles and their clutter-only components (each with 10000 realizations) were generated using the above-described synthesis model and the parameters listed in Table I. The synthesized data were intended to model the received slow-time signal in a normal arterial imaging scenario with minor tissue motion as well as a deep vessel imaging scenario with significant tissue motion, lower transmit frequency, and longer ensemble periods. To gain insights on the Hankel-SVD filter, particular signal realizations were selected from the synthesized data to analyze the characteristics of the principal Hankel components. Also, to assess the filter's performance quantitatively, the Hankel-SVD filter was applied to all the synthesized slow-time signals and their resulting post-filter power was computed. As a benchmark for the post-filter power estimates, the same procedure was repeated with the clutter-only signals. Note that during the analysis, the dimension parameter $P$ of the Hankel-SVD filter was set to five (i.e., $N_{D} / 2$, the maximum possible value), while the two frequency thresholds $f_{\text {thr(clut) }}$ and $\Delta f_{\text {thr }}$ were set, respectively, to $100 \mathrm{~Hz}$ and $50 \mathrm{~Hz}$ (since the clutter spectral range of the two flow scenarios is at most $\pm 95 \mathrm{~Hz}$, as will be shown in Section IV-A).

\section{Performance Measure}

In this study, the post-filter clutter-to-blood signal ratio (CBR) was analyzed at various blood velocities to examine the efficacy of the Hankel-SVD filter quantitatively. This quantity was computed by finding the square-root of the ratio between the average post-filter power of clutteronly signals and that of slow-time signals corresponding to a particular blood velocity. If a filter can effectively suppress clutter, the post-filter CBR should be less than one in linear scale (or be negative in $\mathrm{dB}$ scale) because blood echoes should dominate the filtered slow-time signal.

To facilitate comparative assessment, the above filter analysis was also carried out with an IIR-based (with projection-initialization) clutter-downmixing filter. The 
TABLE I

Simulation Parameters Used in the Clutter Filter Study.

\begin{tabular}{|c|c|c|c|}
\hline Parameter & Arterial Imaging & \multicolumn{2}{|c|}{ Deep Vessel Imaging } \\
\hline \multicolumn{4}{|c|}{ Fixed Machine and Synthesis Parameters } \\
\hline Acoustic speed, $c_{o}$ & \multicolumn{3}{|c|}{$1540 \mathrm{~m} / \mathrm{s}$} \\
\hline F-number, $F_{\text {num }}$ & \multicolumn{3}{|c|}{4} \\
\hline Slow-time ensemble size, $N_{D}$ & \multicolumn{3}{|c|}{10} \\
\hline Dynamic range & \multicolumn{3}{|c|}{14 bits } \\
\hline Number of realizations per dataset & \multicolumn{3}{|c|}{10000} \\
\hline \multicolumn{4}{|c|}{ Manipulated Machine Parameters } \\
\hline Pulse carrier frequency, $f_{o}$ & $5 \mathrm{MHz}$ & & $2 \mathrm{MHz}$ \\
\hline Pulse repetition interval, $T_{\mathrm{PRI}}$ & $0.4 \mathrm{~ms}$ & & $2.0 \mathrm{~ms}$ \\
\hline \multicolumn{4}{|c|}{ Signal Strength Parameters } \\
\hline Clutter-to-blood signal ratio, CBR & \multicolumn{3}{|c|}{$30 \mathrm{~dB}$} \\
\hline Blood-signal-to-noise ratio, BSNR & \multicolumn{3}{|c|}{$10 \mathrm{~dB}$} \\
\hline Average noise strength, $\kappa_{w}$ & \multicolumn{3}{|c|}{$10 \mathrm{~dB}$} \\
\hline \multicolumn{4}{|c|}{ Blood Parameters } \\
\hline Beam-flow angle, $\theta_{b}$ & \multicolumn{3}{|c|}{$60^{\circ}$} \\
\hline Flow velocity, $v_{b}$ & \multicolumn{3}{|c|}{ Varying from 0 to aliasing velocity } \\
\hline \multicolumn{4}{|c|}{ Clutter Parameters } \\
\hline Beam-tissue angle, $\theta_{c}$ & \multicolumn{3}{|c|}{$0^{\circ}$} \\
\hline Maximum tissue velocity, $v_{c, \max }$ & $2 \mathrm{~mm} / \mathrm{s}$ & & $20 \mathrm{~mm} / \mathrm{s}$ \\
\hline Vibration frequency, $f_{\mathrm{vib}}$ & $5 \mathrm{~Hz}$ & & $5 \mathrm{~Hz}$ \\
\hline \multicolumn{4}{|c|}{ Responding Signal Parameters } \\
\hline Ensemble period, $N_{D} T_{\mathrm{PRI}}$ & $4.0 \mathrm{~ms}$ & & $20.0 \mathrm{~ms}$ \\
\hline Aliasing velocity, $v_{\text {alias }}$ & $38.5 \mathrm{~cm} / \mathrm{s}$ & & $19.2 \mathrm{~cm} / \mathrm{s}$ \\
\hline Max instantaneous clutter phase, $\phi_{c, \max }$ & 2.6 radians & & 10.4 radians \\
\hline \multicolumn{4}{|c|}{ Hankel-SVD Filter Parameters } \\
\hline Dimension parameter, $P$ & & 5 & \\
\hline Dominant clutter freq. thr., $f_{\operatorname{thr}(\mathrm{clut})}$ & & $100 \mathrm{~Hz}$ & \\
\hline Clutter stopband size, $\Delta f_{\mathrm{thr}}$ & & $50 \mathrm{~Hz}$ & \\
\hline IIR filter orders (for comparison) & 3 & & 5 \\
\hline
\end{tabular}

IIR filter used was either a third-order (for arterial imaging scenario) or fifth-order (for deep vessel imaging scenario) Chebychev Type-I filter whose nominal cutoff was set arbitrarily (because this parameter's influence on the stopband size is insignificant when using projection initialization). These filter orders were chosen because their effective stopband size (taken as the width between $-60 \mathrm{~dB}$ points in the filter's frequency response) is similar to the cutoff bandwidth of $50 \mathrm{~Hz}$ used by the Hankel-SVD filter. It is worth pointing out that the eigen-regression filter was not examined in these simulations because our signal synthesis model does not generate statistically stationary Doppler ensembles for multiple sample volumes (as needed by the eigen-regression filter to estimate the correlation matrix). Analysis of this filter will be considered in the in vivo imaging case study described in Section V.

\section{Simulation Results and Discussion}

\section{A. Characteristics of Principal Hankel Components}

1. Arterial Imaging Scenario: To illustrate some of the characteristics of the principal Hankel components, a par- ticular realization of the slow-time signal (with an ensemble size of 10) was selected from the data set synthesized with arterial imaging parameters. The blood velocity used to synthesize this signal realization is $10 \mathrm{~cm} / \mathrm{s}$, corresponding to a mean Doppler frequency of $325 \mathrm{~Hz}$. Also, according to the responding parameters listed in Table I (bottom of middle column), this signal realization has a maximum instantaneous clutter phase of 2.6 radians. The theoretical Doppler spectrum for this simulation flow scenario is shown in Fig. 5(a). As can be seen, the clutter phase modulation gives rise to low-frequency impulses that are closely spaced on the Doppler axis between the spectral range of $\pm 40 \mathrm{~Hz}$.

Figs. 5(b) and (c), respectively, show the singular value and mean frequency estimate of the principal Hankel components that correspond to the selected signal realization. From these plots, it can be seen that the first principal component corresponds to the clutter eigen-space based on its distinctly higher singular value magnitude and low mean frequency estimate. Also, the second principal component appears to belong to the flow eigen-space because its mean Doppler frequency estimate is close to the blood's actual mean frequency. The rest of the principal Hankel 
(a) Theoretical Spectrum

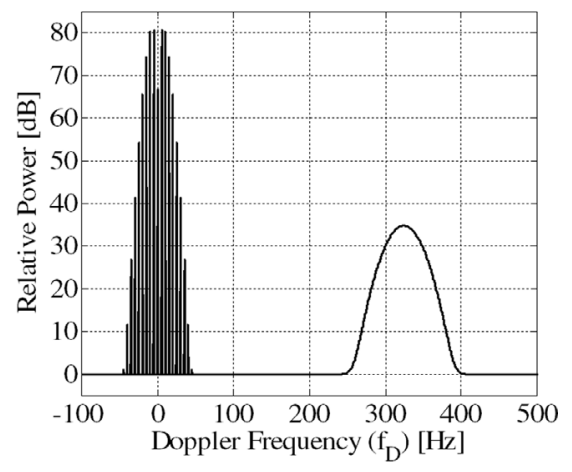

(b) Singular Value Spectrum

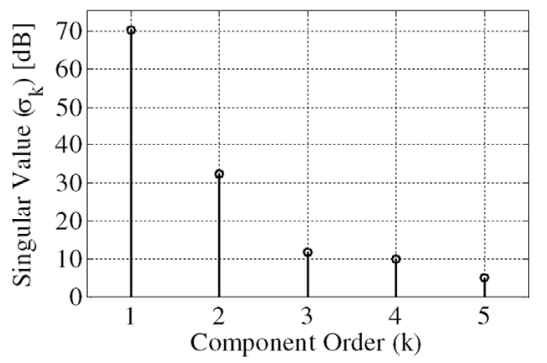

(c) Mean Frequency Estimates

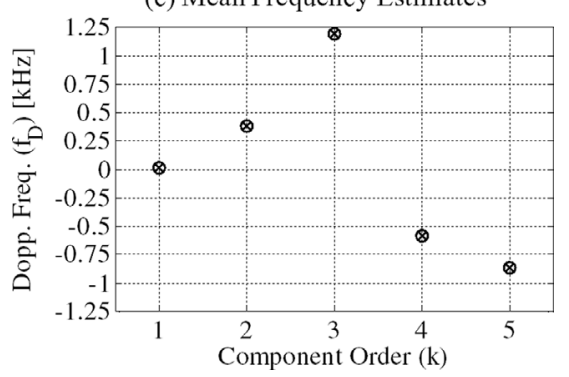

Fig. 5. Characteristics of principal Hankel components for a signal realization in the arterial imaging scenario with $10 \mathrm{~cm} / \mathrm{s}$ blood velocity: (a) the theoretical Doppler spectrum; (b) singular value distribution; (c) mean Doppler frequency estimates (marked as $\otimes$ ).

components appear to correspond to the noise floor since their singular values are relatively small and their mean frequency estimates do not correspond well with the predefined spectral characteristics.

2. Deep Vessel Imaging Scenario: As a comparison to the arterial flow example, a different realization of slowtime signal was selected from the deep vessel imaging data set. For this realization (also with an ensemble size of 10), the blood velocity was $10 \mathrm{~cm} / \mathrm{s}$, corresponding to a mean Doppler frequency of $130 \mathrm{~Hz}$; as well, the signal's maximum instantaneous clutter phase is 10.4 radians (see right column of Table I). An illustration of the slow-time clutter's wideband spectral characteristics is provided in Fig. 6(a), where it can be seen that the low-frequency impulses due to clutter phase modulation (now spanning between $\pm 95 \mathrm{~Hz}$ ) are more significant than the ones in the previous scenario.

Figs. 6(b) and (c) show the corresponding singular values and mean frequency estimates for the selected signal's (a) Theoretical Spectrum

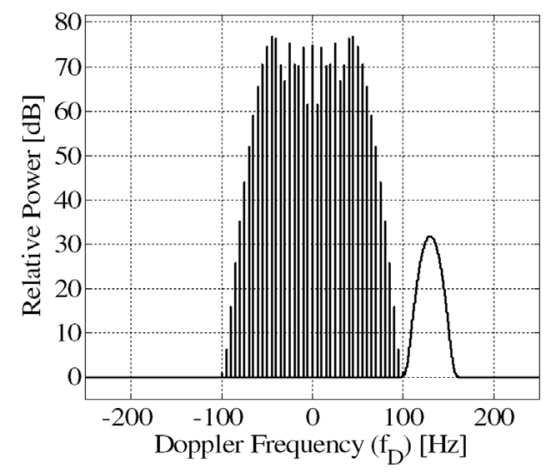

(b) Singular Value Spectrum

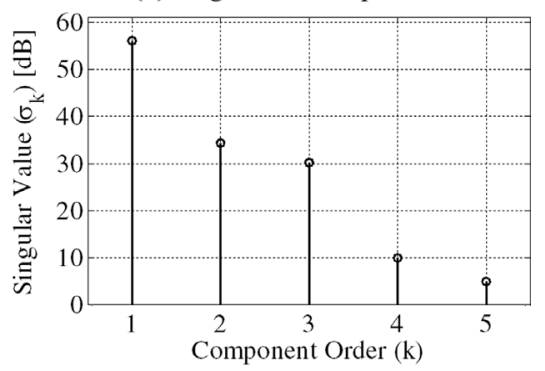

(c) Mean Frequency Estimates

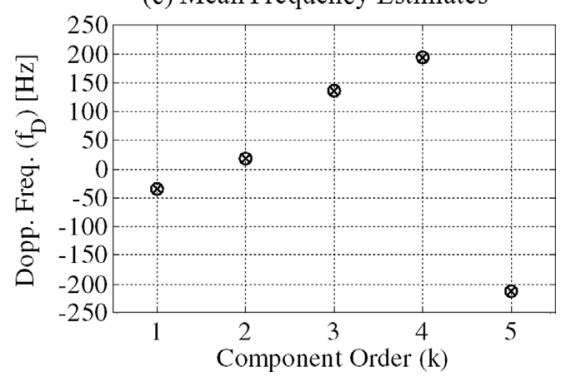

Fig. 6. Characteristics of principal Hankel components for a signal realization in the deep vessel imaging scenario with $10 \mathrm{~cm} / \mathrm{s}$ blood velocity. Descriptions are the same as Fig. 5.

principal Hankel components. It can be seen that the first two principal components have higher singular values and their frequency estimates are within the clutter spectral range; as such, they appear to correspond to the clutter eigen-space. On the other hand, the third principal component appears to belong to the flow subspace because its mean frequency estimate is near the predefined flow Doppler frequency. As for the fourth and fifth principal components, they appear to be describing the noise floor in view of their small singular value magnitudes and their spurious mean frequency estimates.

Based on the results seen in Fig. 5 and Fig. 6, it can generally be concluded that the clutter and blood components are likely to be contained within the first few principal Hankel components corresponding to the slowtime ensemble. Nevertheless, their actual eigen-space dimensions may vary depending on their respective Doppler bandwidths and the random variations inherent in each signal realization. 
(a) Arterial Imaging Scenario

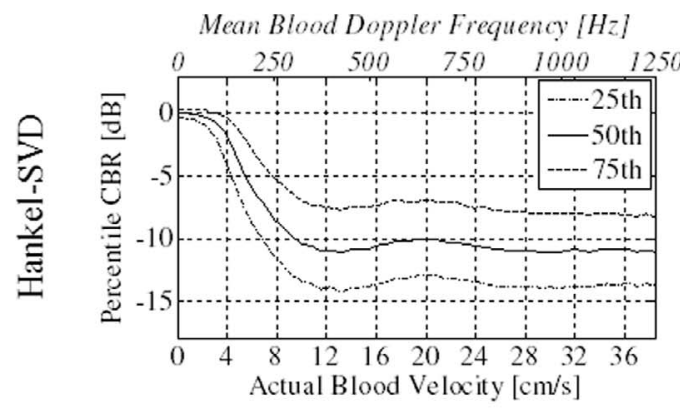

Mean Blood Doppler Frequency $[\mathrm{Hz}]$
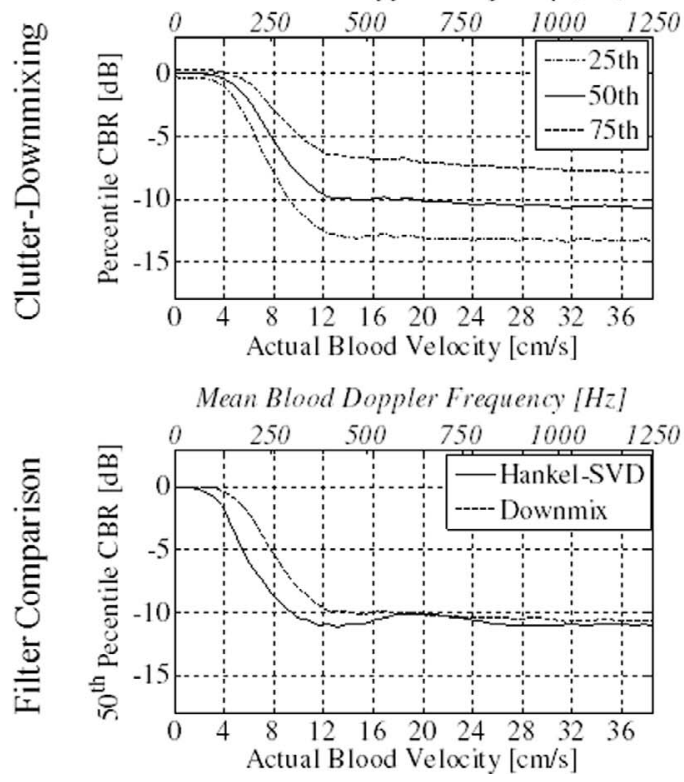

(b) Deep Vessel Imaging Scenario
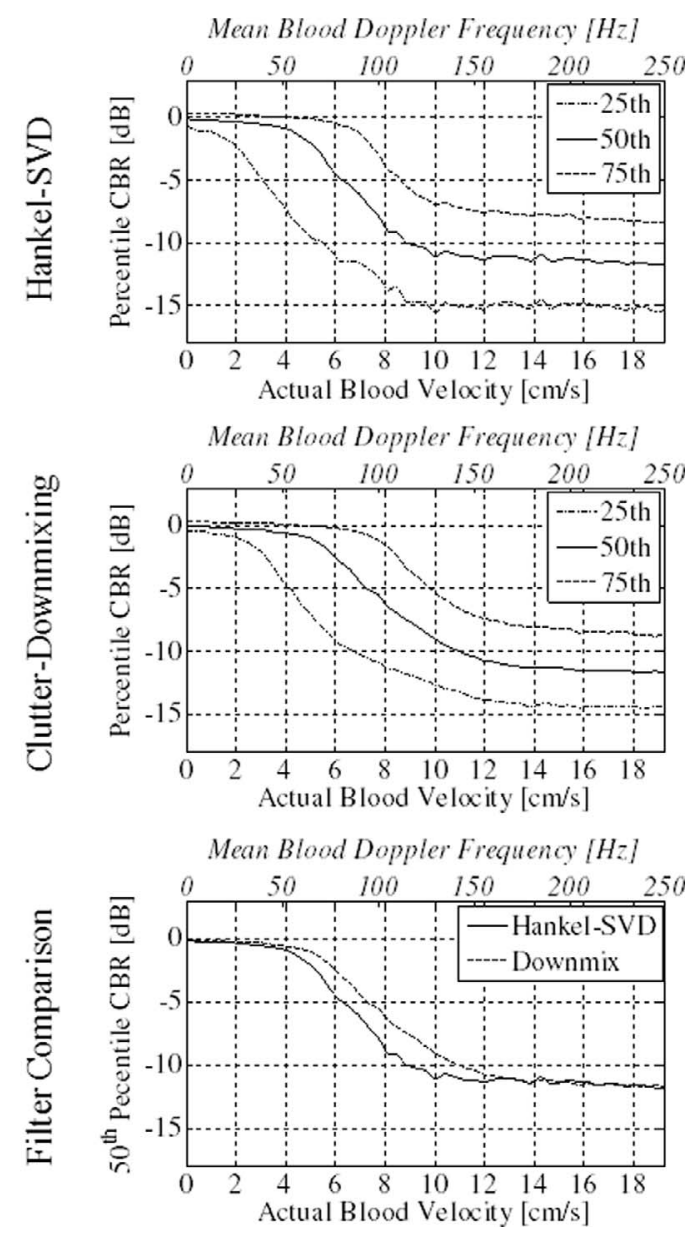

Fig. 7. Post-filter CBR as a function of blood velocity for (a) arterial and (b) deep vessel imaging scenarios. Shown in the top and middle plots, respectively, are the 25th-, 50th-, and 75th-percentile post-filter CBR estimates for the Hankel-SVD filter and the clutter-downmixing filter. Also, a comparison of the 50th-percentile post-filter CBR for the two filters is given in the bottom plots. The filter parameters used to obtain these results are given in Section III-B.

\section{B. Post-Filter Clutter-to-Blood Signal Ratios}

1. Arterial Imaging Scenario: As a quantitative assessment of the new filter's performance in the arterial imaging scenario, the top graph of Fig. 7(a) shows the post-filter CBR as a function of the actual blood velocity for a slowtime ensemble size of 10 samples. The shown results are the 25th-, 50th-, and 75th-percentile post-filter CBR estimates obtained from the 10000 signal realizations synthesized at each blood velocity. Note that various percentile CBR curves are shown in this figure to depict the variations in the distribution of post-filter CBR estimates obtained at each blood velocity. The primary observation to be noted from Fig. 7(a) is that the 50th-percentile (i.e., median) post-filter CBR curves started at a $0 \mathrm{~dB}$ level and then approached the reciprocal of the expected BSNR of $-10 \mathrm{~dB}$ as the flow velocity increases. The $0 \mathrm{~dB}$ trend observed at near-zero flow velocities can be explained by recognizing that the low-velocity blood echoes are likely attenuated concomitantly in the filtering process, so the filtered slow-time signal in these cases tend to share similar form with the filtered clutter-only signal (and in turn, they yield a $0 \mathrm{~dB}$ post-filter $\mathrm{CBR}$ ). On the other hand, the $-10 \mathrm{~dB}$ trend in the higher velocity range can be explained by considering that clutter can more likely be suppressed without distorting high-velocity blood signals, so the filtered clutter-only signal and the filtered slow-time signal in these cases should be dominated, respectively, by white noise and blood echoes (and in turn, they give a post-filter CBR that approaches the expected BSNR's reciprocal). It follows that variations in post-filter CBR values are also expected in the higher velocity range, because the power of filtered clutter-only signals in this range is essentially random noise power that equals to the mean-squared sum of Gaussian random samples.

As a comparison, the middle graph of Fig. 7 shows a similar set of post-filter CBR curves obtained using a clutter-downmixing filter with similar stopband size; also, the bottom graph of Fig. 7 gives a comparison of the median post-filter CBR curves for the two filters. It can be seen that for all the shown post-filter CBR curves (i.e., the 25th-, 50th-, and 75th-percentile curves), the Hankel-SVD filter has a narrower transition region than the clutterdownmixing filter. Because a similar cutoff bandwidth 
TABLE II

Parameters for the In Vivo Flow Imaging Study.

\begin{tabular}{cc}
\hline Parameter & Value \\
\hline Data Acquisition Parameters \\
\hline Transmit pulse frequency & $4 \mathrm{MHz}$ \\
Transmit pulse duration & $0.75 \mu \mathrm{s}(3 \mathrm{cycles})$ \\
Pulse repetition interval & $333 \mu \mathrm{s}$ \\
Slow-time enable size & 10 \\
Beam-flow angle & $\approx 70^{\circ}$ \\
\hline Image Size Parameters \\
\hline Lateral field of view & -11.5 to $+11.5 \mathrm{~mm}$ \\
Number of beam lines & 77 \\
Axial field of view & +5 to $+25 \mathrm{~mm}$ \\
Number of depth samples (per beam) & 115 (non-overlapping) \\
\hline
\end{tabular}

was used for the two filters, this result suggests that the Hankel-SVD filter has a better clutter suppression performance in the case where the slow-time clutter is relatively narrowband with respect to the slow-time sampling rate.

2. Deep Vessel Imaging Scenario: In contrast to the arterial results, Fig. 7(b) shows the post-filter CBR curves in the deep vessel imaging scenario as a function of the true blood velocity. In this scenario, because the slow-time clutter is significantly more wideband in nature [see Fig. 6(a)], the transition regions in all the post-filter CBR curves of both filters are wider. Nevertheless, within the transition region, the Hankel-SVD filter appears to be able to attain the steady-state CBR value at lower velocities (near 8 to $10 \mathrm{~cm} / \mathrm{s}$ ) as compared with the clutter-downmixing filter, which reaches steady-state CBR near 12 to $14 \mathrm{~cm} / \mathrm{s}$. This result is consistent with the findings in the arterial flow scenario. Hence, it seems that the Hankel-SVD filter is generally more capable of suppressing slow-time clutter without concomitantly attenuating the blood signal components.

\section{In Vivo Imaging EXPERIMENT}

To facilitate further evaluation of the Hankel-SVD filter's performance, a frame of in vivo RF color-flow data was acquired using a Philips HDI-5000 scanner (Bothell, WA) that was equipped with an L7-4 linear array probe. The main acquisition parameters are listed in Table II. For this data frame, the field of view corresponded to the cross section of a 25-year-old youth volunteer's carotid arteries (beyond bifurcation) during cardiac systole where arterial wall motion was the most significant [see Fig. 8(a)]. Note that, during the acquisition, the volunteer was asked to hold his breath so that any potential tissue motion due to patient movement can be avoided.

\section{A. Data Analysis Procedure}

The first step in processing the acquired RF data frame is to obtain the analytic slow-time signal of individual sample volumes within the field of view. In our pro- (a) Physical Setting

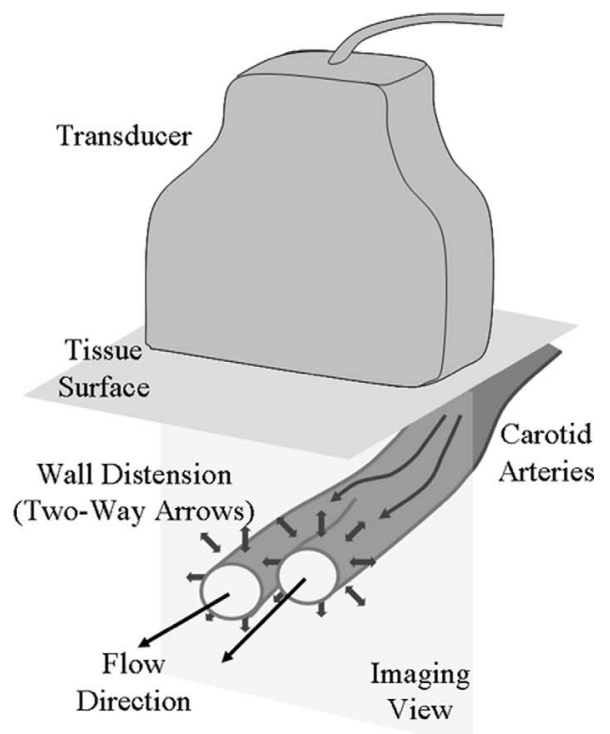

(b) B-mode Image

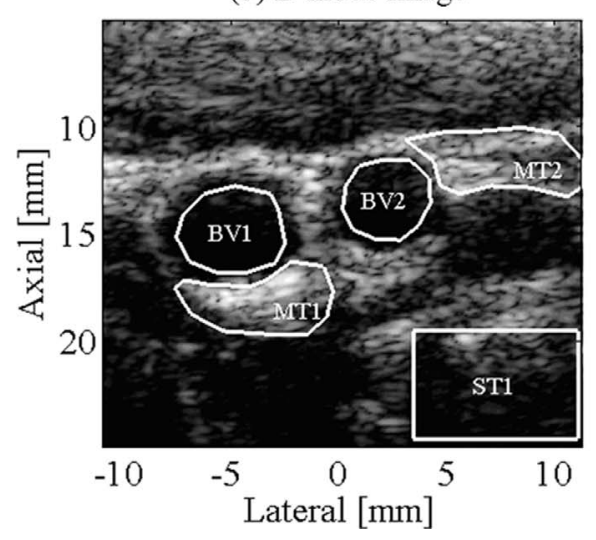

Fig. 8. Graphical overview of an in vivo imaging study on the human carotid arteries (past bifurcation) with tissue motion due to arterial wall distension. In (a), an illustration of the physical setting is shown. In (b), a B-mode image of the imaging view is shown with labeled sections of blood vessel (BV), moving tissue (MT), and static tissue (ST).

cessing, this preprocessing was carried out using an inphase/quadrature $(\mathrm{I} / \mathrm{Q})$ demodulation routine. Once the slow-time ensembles were obtained, they were individually passed into the Hankel-SVD filter to suppress any clutter that may be present, and the resulting filtered signal power was computed. To study the Hankel-SVD filter's sensitivity to blood flow echoes as well as its specificity to clutter, the filtered signal power was compared over different regions of blood vessel, static tissue and moving tissue within the data frame [as depicted in Fig. 8(b)]. It is worth noting that, since the tissue motion in this case study is primarily caused by arterial wall distension during peak systole, the moving tissue sections were selected to be regions adjacent to the blood vessels, while a static tissue section was selected from a region located far away from the blood vessels. In terms of the stopband parameters, the Hankel-SVD filter used for this task had threshold values 
TABLE III

Regional Power for the In Vivo Imaging Study (In DB, with Respect to Unity Reference Level).

\begin{tabular}{lccc}
\hline & & & $\begin{array}{c}\text { Fixed-Rank, } \\
\text { Multi- } \\
\text { Ensemble } \\
\text { Eigen }\end{array}$ \\
\hline Blood vessel 1 (BV1) & 53.2 & 54.2 & 54.5 \\
Blood vessel 2 (BV2) & 53.6 & 53.6 & 54.2 \\
Moving tissue 1 (MT1) & 44.5 & 50.9 & 52.0 \\
Moving tissue 2 (MT2) & 36.4 & 43.6 & 42.6 \\
Static tissue (ST1) & 36.2 & 38.2 & 38.2 \\
\hline
\end{tabular}

of $f_{\mathrm{thr}(\mathrm{clut})}=200 \mathrm{~Hz}$ and $\Delta f_{\mathrm{thr}}=100 \mathrm{~Hz}$. These values were chosen since they yielded high power estimates in the blood vessel regions of the image [the BV1 and BV2 sections in Fig. 8(b)] while giving low ones in the static tissue section.

For comparative assessment, the above filter analysis procedure was repeated for two types of clutter filters: 1) an IIR-based clutter-downmixing filter (2nd-order, projection-initialized), and 2) a fixed-rank multi-ensemblebased eigen-filter (2nd-order clutter dimension). These filter orders were chosen so that they can give blood power estimates that are similar to those provided by the HankelSVD filter. Note that, for the multi-ensemble-based eigenfilter, the slow-time correlation matrix was estimated via ensemble averaging of all slow-time ensembles along the same beam line.

\section{B. Results and Discussion}

Fig. 9 shows the filtered slow-time power maps obtained from all three filters considered in this study. As shown in these images, all three filters have produced power maps that correctly indicated the presence of flow inside the two blood vessels within the imaging view. Nevertheless, as seen by its smaller power estimates outside the blood vessel, the Hankel-SVD filter appears to be able to give a better visualization of the blood vessel regions than the two comparison filters. This observation is further supported by Table III, which shows that the Hankel-SVD filter yielded a larger separation between the power estimates of blood and tissue regions (about $9 \mathrm{~dB}$ difference between the BV2 and MT1 regions, as compared with 2 to $3 \mathrm{~dB}$ for the other two filters considered). Based on these results, it appears that the Hankel-SVD filter is more capable of distinguishing blood flow signals from slow-time clutter when the processors are optimized to provide similar filtered power estimates in the larger blood vessel.

Among the three processors considered in this study, the fixed-rank multi-ensemble-based eigen-filter actually produced power maps with the most distorted view of the blood vessels. This mediocre performance is likely due to two factors associated with the filter's formulation. First, the use of a fixed clutter dimension for the eigen-regression filter may not be appropriate in this case study since the (a) Hankel-SVD Filter

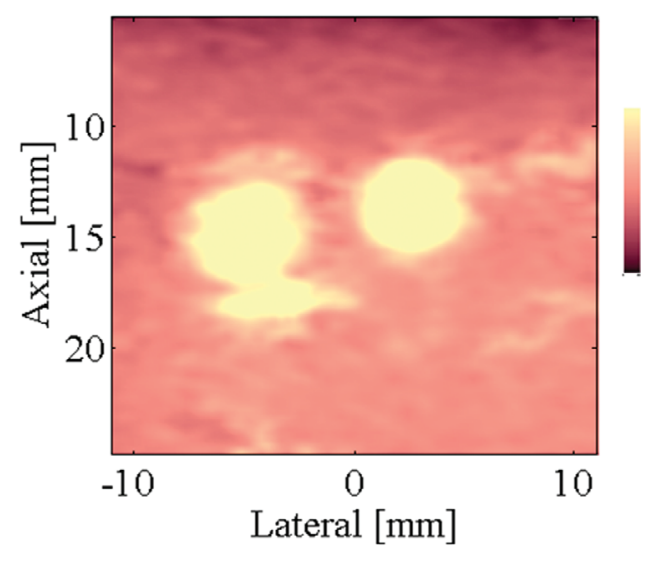

(b) Clutter-Downmixing Filter

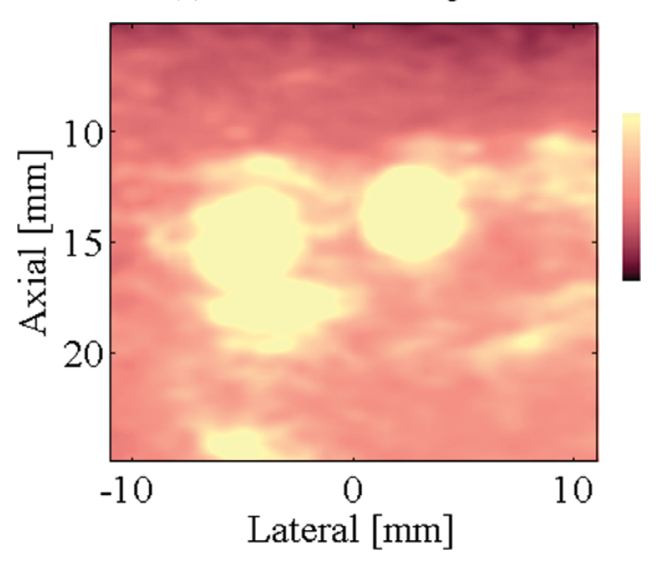

(c) Fixed-Rank Multi-Ensemble Eigen-Filter

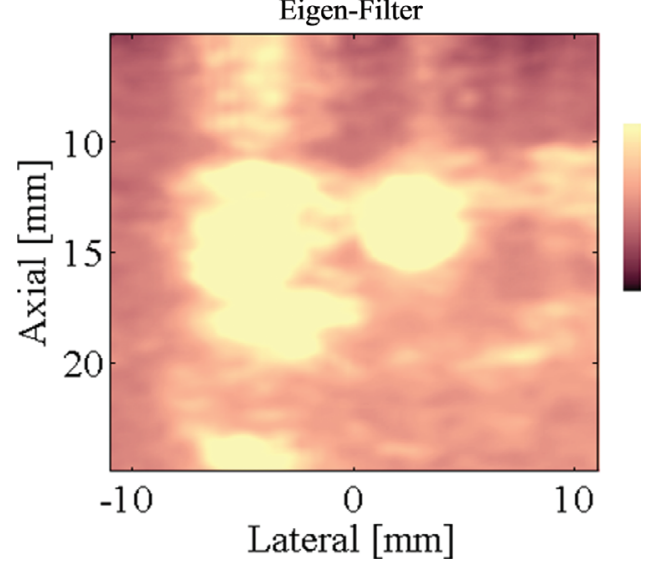

Fig. 9. Filtered slow-time power maps for the in vivo scenario depicted in Fig. 9. Shown in (a), (b), and (c), respectively, are the power maps for the Hankel-SVD filter, clutter-downmixing filter, and the fixed-rank multi-ensemble-based eigen-filter. The dynamic range for these power maps is $30 \mathrm{~dB}$ (from 25 to $55 \mathrm{~dB}$, with respect to a unity reference level). A $5 \times 5$ median-and-mean filter was applied to each power map. 
slow-time clutter characteristics vary spatially over the imaging view. Second, estimation of the slow-time correlation matrix via multi-depth ensemble averaging may be impractical given that clutter statistics are likely to be nonstationary over a depth range of few centimeters. In view of these problems, modifications to the formulation of the multi-ensemble-based eigen-filter seem to be needed for this filter to be effective in the in vivo imaging scenarios where tissue motion varies spatially over the imaging view. Some of these issues have recently been investigated by Lovstakken et al. [13].

\section{Concluding Remarks}

Existing eigen-based clutter filter designs generally require multiple ensembles of slow-time data that are statistically stationary to carry out the eigen-decomposition analysis. To avoid such requirement, this paper has presented a single-ensemble-based eigen-filtering strategythe Hankel-SVD filter-designed to work with the slowtime signal of individual sample volumes. This new approach can generally be beneficial in vascular imaging studies where it is difficult to segment out image regions with slow-time sample volumes that have similar signal characteristics (such as the case where spatially-varying tissue motion is present over a centimeter-scale depth range). Nevertheless, it may not be as effective as existing eigen-based filters for very small ensemble sizes (e.g., when $N_{D}=4$ ) because the Hankel-SVD analysis only decomposes the slow-time signal into, at most, $N_{D} / 2$ orthogonal bases.

A possible limitation of the Hankel-SVD filter is that, during the clutter eigen-space analysis, slow-time clutter is presumed to be contained in the more dominant Hankel components. This assumption is generally valid when clutter is significantly higher in strength as compared with the blood echoes (as is the case in general vascular imaging). However, the assumption would be invalid if the slow-time clutter and the blood echoes are similar in strength (e.g., in high-frequency imaging studies where the blood-scattering strength is higher). In this case, a more advanced algorithm is needed to determine precisely the clutter eigenspace dimension. One possible way of developing such an algorithm is to make use of fuzzy logic and pattern recognition principles [20]. Alternatively, it may be possible to use a multi-modal spectral estimator to first extract several principal frequency estimates in parallel and then determine the clutter eigen-space dimension based on the spectral spread of the estimates.

Aside from its application in ultrasound color flow imaging, the Hankel-SVD filtering technique may be useful to other flow imaging modalities that also involve the suppression of tissue clutter. For instance, this filter may possibly be applied to Doppler optical coherence tomography (OCT) that shares similar flow estimation principles with ultrasound [21]. Indeed, in processing Doppler OCT signals, the proposed filter should be more effective than conventional digital filters because the slow-time clutter is inherently more reflective of the tissue motion owing to the increased velocity resolution provided by OCT scanners.

\section{APPENDIX A \\ Signal Synthesis Equations}

As shown in [19], the slow-time signal of a single scatterer has the following general form for a given transmit frequency $f_{o}$ and acoustic speed $c_{o}$ :

$$
h(n)=b(n) \cdot g(n) \cdot \exp \left\{j 2 \pi f_{o} \frac{2 z(n)}{c_{o}}\right\} .
$$

In the above, $z(n)$ represents the scatterer's displacement with respect to the transducer origin during the $n$th pulse firing, while $b(n)$ and $g(n)$, respectively, represent the beam magnitude and the range gate scaling affecting the scatterer over that particular firing. Based on (A1), we can derive the slow-time signal synthesis equations for blood and clutter by defining the actual forms of $z(n), b(n)$, and $g(n)$.

\section{A. Blood Signals}

For a blood scatterer moving straight at a speed $v_{b}$, its displacement function $z_{b}(n)$ is given by

$$
z_{b}(n)=n T_{\mathrm{PRI}} v_{b} \cos \theta_{b},
$$

where $T_{\mathrm{PRI}}$ is the pulse repetition period and $\theta_{b}$ is the beam-flow angle. By substituting this relation into (A1), we can see that a blood scatterer gives rise to a slow-time signal of this form:

$$
\begin{aligned}
h_{b}(n) & =b(n) \cdot g(n) \cdot \exp \left\{j 2 \pi f_{o}\left[\frac{2 v_{b} \cos \theta_{b} n T_{\mathrm{PRI}}}{c_{o}}\right]\right\} \\
& =b(n) \cdot g(n) \cdot \exp \left\{j 2 \pi f_{D(b)} n T_{\mathrm{PRI}}\right\},
\end{aligned}
$$

where $f_{D(b)}=2 v_{b} f_{o} \cos \left(\theta_{b}\right) / c_{o}$ is the Doppler frequency. Note that, at large beam-flow angles, the scatterer crosses the sample volume through the effective boundaries of the ultrasound beam, and thus $b(n)$ in (A3) can be assumed as a windowing function while $g(n)$ can be treated as a constant. By further assuming that $b(n)$ is a Gaussian window, (A3) can then be seen as being equal to the blood signal template in (12).

\section{B. Clutter}

In contrast to blood, tissue tends to pivot and move in a quasicyclic manner similar to a spring oscillator. As such, its displacement function $z_{c}(n)$ essentially takes on an alternating form rather than a linear form like that for blood. If the tissue scatterer moves purely in a sinusoidal fashion (i.e., like a harmonic oscillator), then $z_{c}(n)$ has the following form:

$$
z_{c}(n)=\left\{z_{c, \max } \cos \theta_{c}\right\} \sin \left(2 \pi f_{\mathrm{vib}} n T_{\mathrm{PRI}}\right),
$$


where $z_{c, \max }$ represents the maximum displacement, $\theta_{c}$ is the beam-tissue angle, and $f_{\text {vib }}$ is the oscillation frequency. By taking the derivative of $z_{c}(n)$, we can define a tissue velocity function $v_{c}(n)$ equal to

$$
v_{c}(n)=\frac{d}{d n}\left[z_{c}(n)\right]=\left\{v_{c, \max } \cos \theta_{c}\right\} \cos \left(2 \pi f_{\mathrm{vib}} n T_{\mathrm{PRI}}\right),
$$

where $v_{c, \text { max }}=2 \pi f_{\mathrm{vib}} z_{c, \text { max }}$ is the maximum tissue velocity. By incorporating the above relations into (A1), the slow-time signal for a tissue scatterer can then be expressed as

$$
\begin{aligned}
h_{c}(n)= & b(n) \cdot g(n) \\
& \cdot \exp \left\{j\left[\frac{2 f_{o} v_{c, \max } \cos \theta_{c}}{c_{o} f_{\mathrm{vib}}}\right] \sin \left(2 \pi f_{\mathrm{vib}} n T_{\mathrm{PRI}}\right)\right\} \\
= & b(n) \cdot g(n) \cdot \exp \left\{j \phi_{c, \max } \sin \left(2 \pi f_{\mathrm{vib}} n T_{\mathrm{PRI}}\right)\right\},
\end{aligned}
$$

where $\phi_{c, \max }$ is the maximum instantaneous clutter phase as given in (11). Assuming that the tissue movement is not beyond the effective boundaries of the sample volume, both $b(n)$ and $g(n)$ in the above relation can be regarded as constants, and consequently (A6) has the same form as the clutter waveform template in (10).

\section{ACKNOWLEDGMENTS}

We wish to thank Dr. Matthew Bruce (Philips Medical Systems) and Dr. Peter Burns (Medical Biophysics Department, University of Toronto) for providing valuable advice on our in vivo case study. We are also very grateful to Dr. Wayne Johnston (Vascular Surgery Division, Toronto General Hospital) and the anonymous reviewers for their constructive feedback.

\section{REFERENCES}

[1] S. Bjaerum, H. Torp, and K. Kristoffersen, "Clutter filter design for ultrasound color flow imaging," IEEE Trans. Ultrason., Ferroelect., Freq. Contr., vol. 49, pp. 204-216, 2002.

[2] L. Thomas and A. Hall, "An improved wall filter for flow imaging of low velocity flow," in Proc. IEEE Ultrason. Symp., 1994, pp. 1701-1704.

[3] P. J. Brands, A. P. G. Hoeks, and R. S. Reneman, "The effect of echo suppression on the mean velocity estimation range of the RF cross-correlation model estimator," Ultrasound Med. Biol., vol. 21, pp. 945-959, 1995.

[4] A. C. H. Yu, K. W. Johnston, and R. S. C. Cobbold, "Frequencybased signal processing in ultrasound color flow imaging," Can. Acoust., vol. 35, no. 2, pp. 11-23, 2007.

[5] L. A. F. Ledoux, P. J. Brands, and A. P. G. Hoeks, "Reduction of the clutter component in Doppler ultrasound signals based on singular value decomposition: A simulation study," Ultrason. Imag., vol. 19, pp. 1-18, 1997.

[6] S. Bjaerum, H. Torp, and K. Kristoffersen, "Clutter filters adapted to tissue motion in ultrasound color flow imaging," IEEE Trans. Ultrason., Ferroelect., Freq. Contr., vol. 49, pp. 693-704, 2002

[7] D. E. Kruse and K. W. Ferrara, "A new high resolution color flow system using an eigendecomposition-based adaptive filter for clutter rejection," IEEE Trans. Ultrason., Ferroelect., Freq. Contr., vol. 49, pp. 1384-1399, 2002.
[8] C. Kargel, G. Hobenreich, B. Trummer, and M. F. Insana, "Adaptive clutter rejection filtering in ultrasonic strain-flow imaging," IEEE Trans. Ultrason., Ferroelect., Freq. Contr., vol. 50, pp. 824-835, 2003.

[9] C. M. Gallippi and G. E. Trahey, "Adaptive clutter filtering via blind source separation for two-dimensional ultrasonic blood velocity measurement," Ultrason. Imag., vol. 24, pp. 193-214, 2002.

[10] C. M. Gallippi, K. R. Nightingale, and G. E. Trahey, "BSS-based filtering of physiological and ARFI-induced tissue and blood motion," Ultrasound Med. Biol., vol. 29, pp. 1583-1592, 2003.

[11] A. J. van der Veen, E. F. Deprettere, and A. L. Swindlehurst, "Subspace based signal analysis using singular value decomposition," Proc. IEEE, vol. 81, pp. 1277-1308, 1993.

[12] M. W. Y. Poon, R. H. Khan, and S. Le-Ngoc, "A singular value decomposition (SVD) based method for suppressing ocean clutter in high frequency radar," IEEE Trans. Signal Processing, vol. 41, pp. 1421-1425, 1993.

[13] L. Lovstakken, S. Bjaerum, K. Kristoffersen, and H. Torp, "Realtime adaptive clutter rejection filtering in color flow imaging using power method iterations," IEEE Trans. Ultrason., Ferroelect., Freq. Contr., vol. 53, pp. 1597-1608, 2006.

[14] C. Kasai, K. Namekawa, A. Koyano, and R. Omoto, "Real-time two-dimensional blood flow imaging using an autocorrelation technique," IEEE Trans. Sonics Ultrason., vol. 32, pp. 458-464, 1985.

[15] A. C. H. Yu, "Eigen-based signal processing methods for ultrasound color flow imaging," Ph.D. dissertation, University of Toronto, Toronto, ON, Canada, 2007.

[16] G. H. Golub and C. F. van Loan, Matrix Computations. 3rd ed. Baltimore, MD: Johns Hopkins University Press, 1996.

[17] A. Heimdal and H. Torp, "Ultrasound Doppler measurements of low velocity blood flow: Limitations due to clutter signals from vibrating muscles," IEEE Trans. Ultrason., Ferroelect., Freq. Contr., vol. 44, pp. 873-881, 1997.

[18] K. Kristoffersen and B. A. J. Angelsen, "A time-shared ultrasound Doppler measurement and 2-D imaging system," IEEE Trans. Biomed. Eng., vol. 35, pp. 285-295, 1988.

[19] A. C. H. Yu, A. H. Steinman, and R. S. C. Cobbold, "Transit time broadening in pulsed Doppler ultrasound: A generalized amplitude modulation model," IEEE Trans. Ultrason., Ferroelect., Freq. Contr., vol. 53, pp. 530-541, 2006.

[20] B. Hu and R. G. Gosine, "A new eigenstructure method for sinusoidal signal retrieval in white noise: Estimation and pattern recognition," IEEE Trans. Signal Processing, vol. 45, pp. 30733083, 1997.

[21] V. X. Yang, M. L. Gordon, B. Qi, J. Pekar, S. Lo, E. Seng-Yue, A. Mok, B. C. Wilson, and I. A. Vitkin, "High speed, wide velocity dynamic range Doppler optical coherence tomography (Part I): System design, signal processing, and performance," Opt. Express, vol. 11, pp. 794-809, 2003.

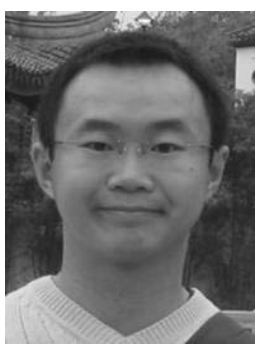

Alfred C. H. Yu (S'99-M'07) was born in Hong Kong in 1980. He received his B.Sc. degree in electrical engineering from the University of Calgary, AB, Canada, in 2002. He then completed his M.A.Sc. and Ph.D. degrees in electrical engineering (biomedical stream) from the University of Toronto, ON, Canada, in 2003 and 2006, respectively. In the summer of 2005 , he was a research intern at Philips Research, Briarcliff Manor, NY, United States.

For his graduate work, Alfred studied the theoretical aspects of eigen-based signal processing strategies for Doppler ultrasound and color-flow imaging. He also worked on the modeling of Doppler ultrasound signals and Doppler spectral broadening. His graduate work was funded by the Canada Graduate Scholarship program from the Natural Sciences and Engineering Research Council of Canada.

Alfred is currently a postdoctoral research fellow and honorary assistant professor in the Department of Electrical and Electronic Engineering at the University of Hong Kong. His research interests are broadly centered around the design aspects of ultrasound systems and devices. He is also interested in studying the fundamentals and physics of ultrasound. 


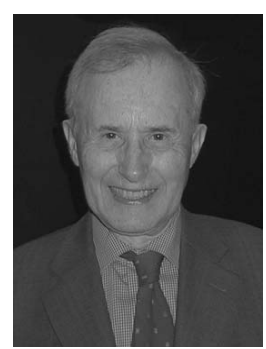

Richard S. C. Cobbold (M'59-LM'96) received the B.Sc. degree in physics from Imperial College, University of London in 1956, and obtained the M.Sc. and Ph.D. degrees from the University of Saskatchewan, Canada in 1961 and 1965, respectively. Since 1966, he has been with the Institute of Biomaterials and Biomedical Engineering, University of Toronto, where he was director from 1974 through to 1983 . He is currently professor emeritus in the institute and in the Department of Electrical and Computer Engineering. His research interests center around the fundamental aspects of ultrasound, Doppler ultrasound, and its application for vascular assessment. He is the author of three graduate-level books, a number of research papers, and several book chapters. His most recent book, Foundations of Biomedical Ultrasound, was published by Oxford University Press in 2006. He was elected a Fellow of the Royal Society of Canada in 1976 and was appointed Canada-UK Rutherford lecturer in 1995. 